\title{
Direct Laser Write (DLW) as a Versatile Tool in Manufacturing Templates for Imprint Lithography on Flexible Substrates
}

\author{
Marius G. Ivan ${ }^{* a}$, Jean-Baptiste Vaney ${ }^{\mathrm{a}}$, Dick Verhaart ${ }^{\mathrm{b}}$, and Erwin R. Meinders ${ }^{\mathrm{a}}$ \\ ${ }^{1}$ Holst Centre/ TNO, High Tech Campus 31, PO Box 8550, 5656 AE Eindhoven, The Netherlands \\ ${ }^{2}$ Singulus Mastering B.V., Marinus v. Meelweg 2, 5657 EN Eindhoven, The Netherlands
}

\begin{abstract}
A computer-controlled laser beam recorder with a wavelength of $405 \mathrm{~nm}$ has been employed for patterning the deposited resist with feature sizes varying from a few hundreds of nanometers to tens of micrometers. Four inch silicon templates for hot embossing source/ drain electrodes and metallic circuit for a disposable biosensor were obtained. SEM and optical microscopy reveal accurate transfer of developed photoresist structures into the underlying silicon wafer after plasma dry etching. Etch depths between $100-600 \mathrm{~nm}$ were obtained on the templates, and were further transferred into the imprinted plastic substrate and the metallic layer.
\end{abstract}

Keywords: direct laser write, nanoimprint lithography, plastic electronics, hot embossing, silicon template, flexible substrate

\section{INTRODUCTION}

The huge potential of the plastic electronics market has driven the research in this field, allowing for impressive developments over the past few years.[1,2] First plastic electronics products, like rollable displays, smart cards, photovoltaics, and book readers have been already commercialized. While increased functionality, like bendability, light weight and robustness, has fuelled the market introduction of these novel plastic electronics products, the mass market adaptation of these applications will be highly dictated by the ability to reduce manufacturing costs. The introduction of large-area manufacturing methods, such as roll-to-roll or sheet-to-sheet processing, will drive throughput and cost reduction. A clear challenge of roll-to-roll processing is the dimensional instability of the plastic substrate. In the particular in case of transistor devices, high alignment accuracies are required.[3] Imprint lithography is one of the obvious patterning technologies suitable for roll-to-roll processing and with high-accuracy alignment potential.[4-6] Nanoimprint lithography (NIL) has been around for more than a decade, and it has developed tremendously in the past years, allowing precision patterning of various layers at nanometer and micrometer scale.[2] More recently, imprint lithography has been positioned as a strong candidate for manufacturing transistors on flexible substrates.[7-11] Due to its high throughput, NIL has been detected as a technology for fabrication of low cost plastic electronics in roll-to-roll fashion.[1, 4-6] In NIL the patterns are formed by pressing a template against a layer of thermoplastic or photosensitive material, which plays the role of the photoresist in optical lithography. In both hot embossing (HE) and step-and flash imprint lithography (SFIL) the pattern is transferred by pressing a stamp against the polymer which is cured either by heating or by UV treatment. While HE requires relatively high temperatures and pressure, SFIL is performed at room temperature and moderate pressures. Fabrication of stamps or templates for imprint lithography requires precise patterning, and it has traditionally been done by mask-based optical lithography and e-beam lithography.

The need for precise and fast methods for fabrication of two- and three-dimensional structures has led to the widespread use of lasers in patterning functional layers of organic and inorganic materials over any potential substrate.[12] Upon interaction with the material, the laser beam may induce photo-thermal reactions, such as ablation, sintering, melting, phase-transformations, photopolymerization, polymer chain scission, etc. Direct laser write (DLW) is a patterning technique that allows easy transferring of a circuit layout onto a functional layer by scanning a laser beam over the substrate.[13] Various techniques of DLW have been developed, with applications in fabrication of optical elements[14, 15], polymer field effect transistors[16], microbatteries and sensors.[17] The use of lasers for patterning resists with

* marius.ivan@tno.nl, ; phone +31 40277 4348; www.holstcentre.com

Alternative Lithographic Technologies, edited by Frank M. Schellenberg, Bruno M. La Fontaine Proc. of SPIE Vol. 7271, 72711S · (C) 2009 SPIE · CCC code: 0277-786X/09/\$18 · doi: 10.1117/12.814481 
application in lithography has been introduced more than two decades ago.[18] The advantage that DLW offers over mask-based lithography is the absence of the projection mask, an expensive part of the projection system in any optical lithography tool. The advantages over an e-beam lithography system are the cost of operation, writing time, and system stability. The laser beam is controlled by a computer, and the pattern to be written can be easily altered at any time, allowing for convenient manufacturing of prototype devices.[19] The downside is the low writing speed, considering that one laser beam is typically used, however the writing time is still considerably shorter than in case of e-beam writing lithography. The minimum feature size depends on the wavelength and the numerical aperture of the lens and can easily reach $150 \mathrm{~nm}$ in case of diffractive-limited optics and visible lasers. For plastic electronics the size of the patterned features is typically in the micrometer regime. A combination between the versatility and ease of patterning offered by DLW and high throughput patterning of plastic substrates with NIL appears as an appealing solution to manufacturing large area flexible electronics on plastic substrate.

The goal of the present study was to show the adaptability of a laser beam recorder (LBR) used for manufacturing CD and DVD masters into a tool for manufacturing silicon (Si) templates with patterns having various sizes and shapes suitable for imprint lithography. A key challenge was a coordinate transformation conversion to pattern rectangular features on a rotational system. Si templates for HE lithography having patterns with large and small feature sizes $(<1.0$ $\mu \mathrm{m}->50.0 \mu \mathrm{m})$, and with various depths, between $100-600 \mathrm{~nm}$, were developed with the developed tool. Templates were tested in imprint experiments on poly(ethyleneterephthalate) (PET) substrates. At the same time we demonstrate that DLW is a very suitable technology for manufacturing imprint templates without the need of a reticle, traditionally used in mask-based optical lithography, or an expensive e-beam pattern writer.

\section{LASER BEAM RECORDER}

A Singulus Laser Beam Recorder (LBR), developed for optical disc mastering, was employed in this study. Conventionally, the LBR is used to pattern a spiral of marks or a continuous groove into a photoresist layer. In a subsequent process, the recorded spiral-shaped pattern is transferred in a metal stamp via an electroplating process and used for mass-replication of optical discs. The spiral-shaped pattern is recorded via a single laser spot that is focused onto a rotating substrate, and slowly moved outwards in the radial direction. A schematic of the writing principle is given in Figure 1. The blue solid state laser source generates laser light at a wavelength of $405 \mathrm{~nm}$. The laser beam is shaped and focused through an objective lens with a numerical aperture of 0.9 , yielding an approximate half intensity width of the focused laser spot of $225 \mathrm{~nm}$.

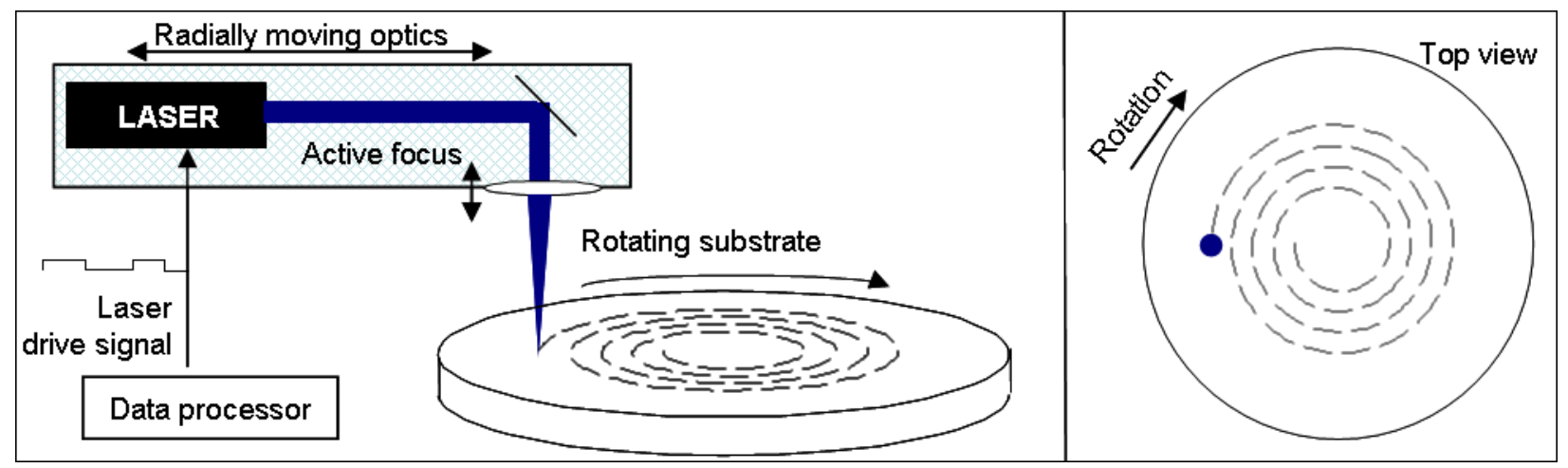

Fig. 1. Schematic representation of Laser Beam recorder writing principle and signal path: the image is written by combining the rotational movement of the substrate with the movement of the laser beam along the $\mathrm{x}$ direction.

Spot size may be increased by reducing the numerical aperture. A second red laser beam is used to control the focus of the objective lens.

The mechanical design (i.e. a heavy stone base, the rotation and translation systems are mounted on high precision air bearings) and the specially designed rotation, translation and focus servo systems guarantee $5 \mathrm{~nm}$ accuracy for the relative spot position. The absolute spot position is $100 \mathrm{~nm}$. The resolution in the radial (track) direction (number of pixels per revolution) is determined by the employed spot size and by the required accuracy of the recorded test patterns. 
For high-resolution patterning, the track pitch/pixels size is made smaller than the spot size to ensure smooth boundaries on objects that are at skew angles to the recording direction, as objects defined on a rectangular grid will necessarily be on the larger part of the wafer area. Prior to recording, the image is converted to polar coordinates. Dedicated software was developed to address the file complexity. The converted images were recorded using a Singulus data processor, type ACE 250, which controls and mutually synchronizes the recording laser, rotation and translation. A flowchart of the steps required for image formation is presented in Fig. 2.

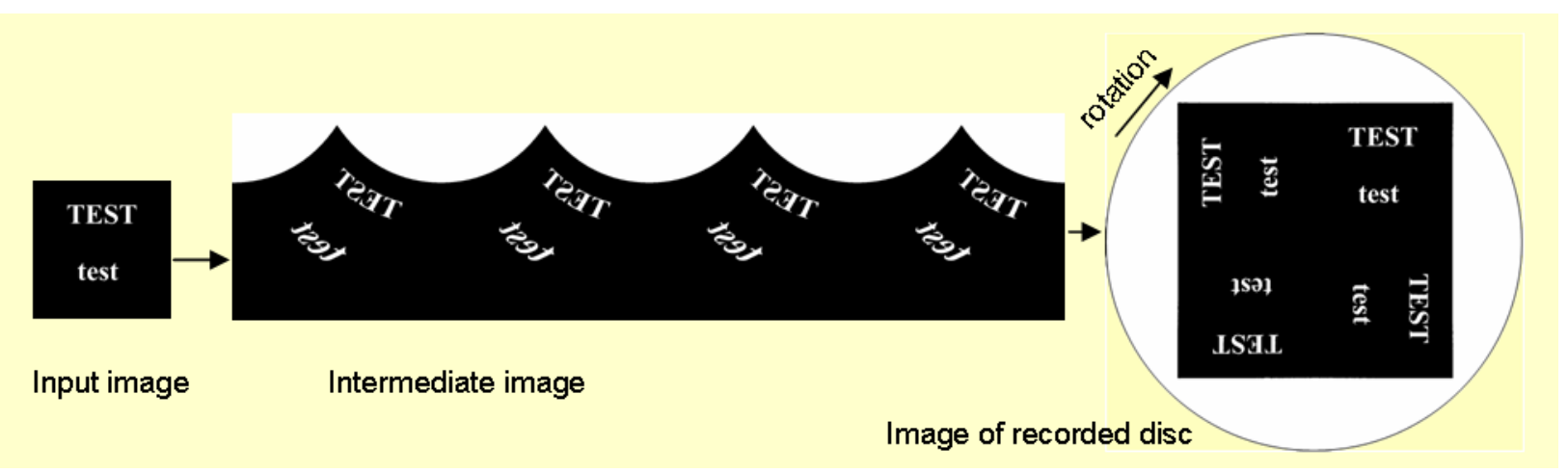

Fig. 2. Schematic representation of data processing and recording, with the sequence of image transformations from the input image in Cartesian coordinates to the intermediate image in polar coordinates, and the image recorded on the disc.

The typical rotation frequency is $50 \mathrm{~Hz}$. To ensure a constant energy density across the entire recording area, the laser power was adjusted proportional to the radius since the substrate was recorded at constant angular velocity. A typical laser power density of $0.7 \mu \mathrm{J} / \mathrm{cm}$ was used for the recordings presented in this publication. With a track pitch of $200 \mathrm{~nm}$, the calculated energy density is $35.5 \mathrm{~mJ} / \mathrm{cm}^{2}$. The recording time depends on the recorded area and amounts to about 2060 minutes for 4 "wafers.

\section{TEMPLATE MANUFACTURING}

By employing DLW at $405 \mathrm{~nm}$ we aimed at manufacturing Si templates bearing patterns with various shapes and dimensions. These templates are to be used in patterning functional layers for manufacturing of transistors on flexible substrates. A key challenge was to fine tune all the exposure parameters such that the different sizes were accommodated on the same template within one exposure experiment.

The photoresist employed was a i-line diazonaphthoquinone/novolak positive resist, HPR 504, spin-coated on TMSDEA vapour primed 4" silicon wafers. AFM and SEM measurements revealed a resist thickness after development of 1.30 $\mu \mathrm{m}$. This thickness was slightly less than that of the exposed film due to dark erosion, which occurs during development. The thermal treatment of the resist involved post baking at $90^{\circ} \mathrm{C}$ for two minutes and post exposure baking at $120^{\circ} \mathrm{C}$ for four minutes. Optical microscope and SEM images of the developed resist pattern and the etched Si template were compared to the imprinted feature sizes.

\subsection{Test patterns}

The patterns to be imprinted were designed with graphics software. The energy was systematically varied with increments of $5 \%$ relative to a nominal value, namely $+5,+2,0,-5,-10,-15,-20$, and $-25 \%$.

The desired patterns contain lines and spaces of different widths. In Fig. 3 the targeted space (exposed areas) was 3.0 $\mu \mathrm{m}$, the islands (unexposed areas) were $27.0 \mu \mathrm{m}$. Even with the lowest incident energy the resist was overexposed, the measured space being $\sim 10 \%$ larger than the intended dimension. However, the clear trend in dimensions as a function of the incident energy allowed an easy detection of the suitable energy settings which allow obtainment of feature sizes with the targeted dimension. The completely cleared out photoresist results in formation of well defined structures, as evidenced by the SEM images. 


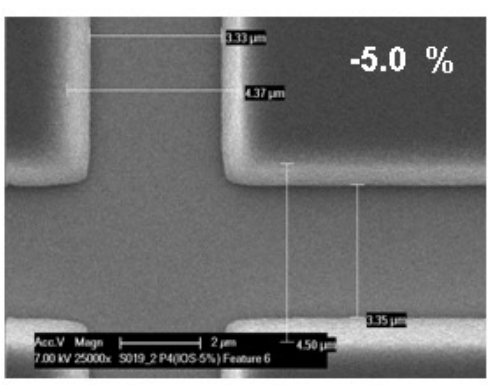

$3.33 \mu \mathrm{m}$

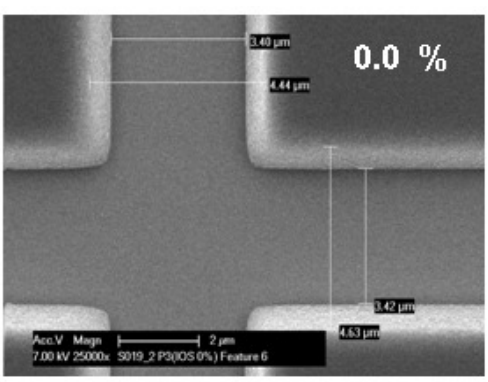

$3.40 \mu \mathrm{m}$

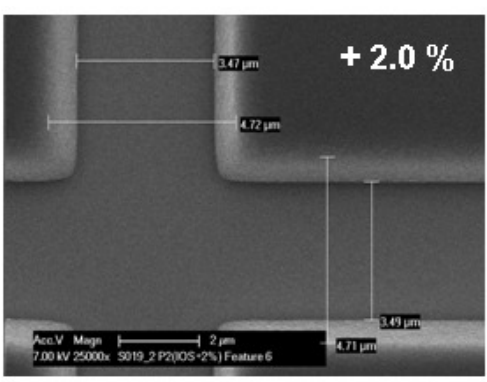

$3.47 \mu \mathrm{m}$

Fig. 3. Exposed and developed photoresist profiles for three different energy doses at $405 \mathrm{~nm}$ and development. The target feature sizes were $30 \mu \mathrm{m}$ pitch with $3.0 \mu \mathrm{m}$ spaces; energy was varied with $-5 \%, 0 \%$, and $+2.0 \%$ relative to the nominal energy. The measured spaces were $3.33,3.40$, and $3.47 \mu \mathrm{m}$ respectively.

To further test the capabilities of writing structures with small dimensions, we exposed $1.0 \mu \mathrm{m}$ lines and $1.0 \mu \mathrm{m}$ spaces on the same wafer with features sizes as large as $50 \mu \mathrm{m}$. The SEM images of patterns written with $-5 \%, 0 \%$ and $+2 \%$ energy relative to the nominal dose show overexposure at nominal and $+2 \%$ energy, while at $-5 \%$ the obtained feature size is very close to the targeted value. The images obtained with the lowest incident energy show a difference of only $2 \%$ from the targeted value of $1.0 \mu \mathrm{m}$, see Fig. 4 . The pitch for these exposures was $125 \mathrm{~nm}$. The slight waviness observed in the developed resist structures is caused by a combination of factors: combination of rotational and $\mathrm{x}$ movement of the substrate and laser beam respectively, and the size of the pixel in the input image. The pixel size was $200 \mathrm{~nm}$, but pixel size as small as $100 \mathrm{~nm}$ could be used as well.

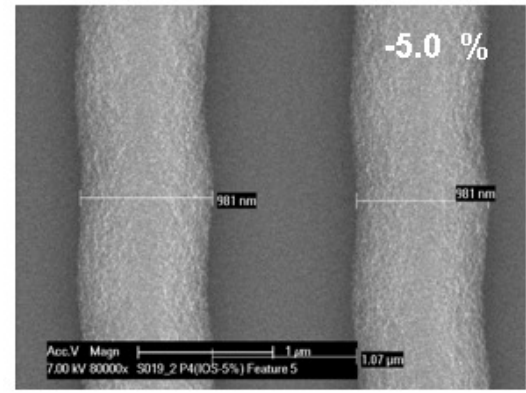

$981 \mathrm{~nm}$

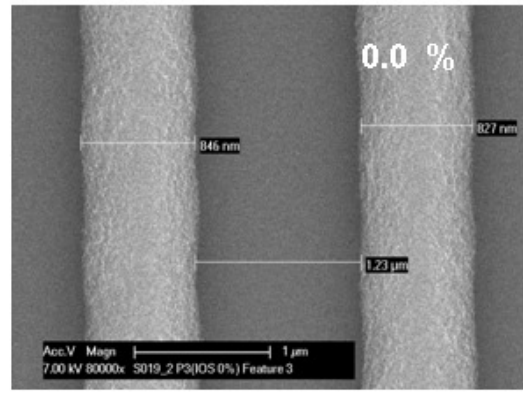

$846 \mathrm{~nm}$

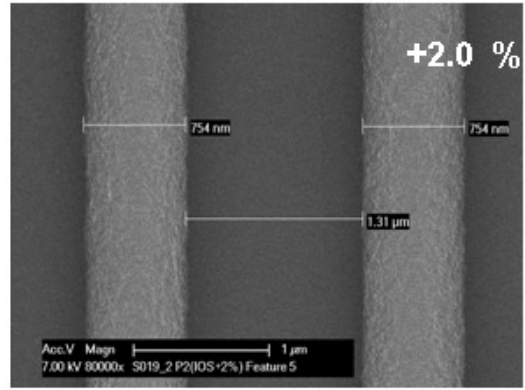

$754 \mathrm{~nm}$

Fig. 4. Photoresist profile following exposure to three different energy doses at $405 \mathrm{~nm}$ and development; target feature sizes were $2.0 \mu \mathrm{m}$ pitch with $1.0 \mu \mathrm{m}$ lines; energy was varied with $-5 \%$ and $0 \%$, and $+2.0 \%$ relative to the nominal energy, and the measured line widths were 981,846 , and $754 \mathrm{~nm}$ respectively.

\subsection{Source/ Drain electrodes}

DLW with optimized exposure parameters was employed to obtain Si templates with various layouts of source and drain electrodes having dimensions from $<1.0 \mu \mathrm{m}$ to $>50 \mu \mathrm{m}$ on the same 4" Si template, see Fig. 5-8. Top views of etched profiles on Si templates are displayed in Fig. 5. S/D with $1.0 \mu \mathrm{m}$ feature sizes and $1.0 \mu \mathrm{m}$ channel length were etched on Si template with a targeted depth of $200 \mathrm{~nm}$, Fig 5A. Channel length of $1.25 \mu \mathrm{m}$ was obtained for Si templates with targeted depths of 400 and $600 \mathrm{~nm}$ respectively, Fig $5 \mathrm{~B}, \mathrm{C}$.

HPR 504 i-line photoresist was used for patterning. The resist patterns were transferred into the silicon substrate via dry reactive ion etching (DRIE) in $\mathrm{CF}_{4}$ plasma atmosphere under controlled conditions, Fig. 6 . The etching times were adjusted to obtain targeted depths of 200, 400, and $600 \mathrm{~nm}$. Examples of etched Si substrates are given in Fig 5-7. 

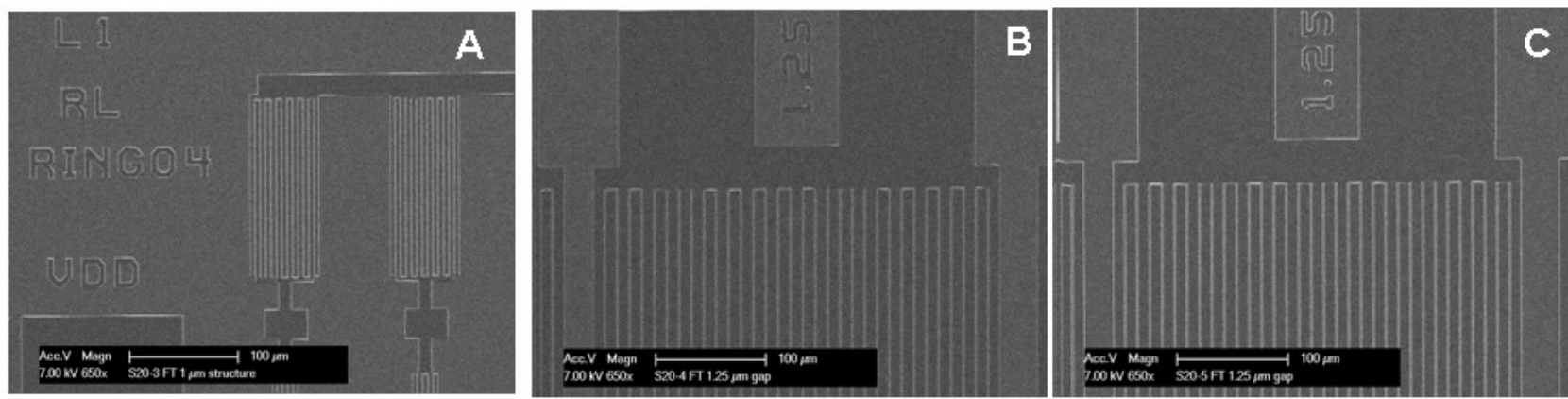

Fig. 5 SEM images of $1.0 \mu \mathrm{m} \mathrm{S} / \mathrm{D}$ electrodes with $1.0 \mu \mathrm{m}$ channel length after etching Si templates for $200 \mathrm{~nm}$ depth; source/ drain electrodes with $1.25 \mu \mathrm{m}$ channel length and etch depths of $400 \mathrm{~nm}$ (B) and $600 \mathrm{~nm}(\mathrm{C})$.
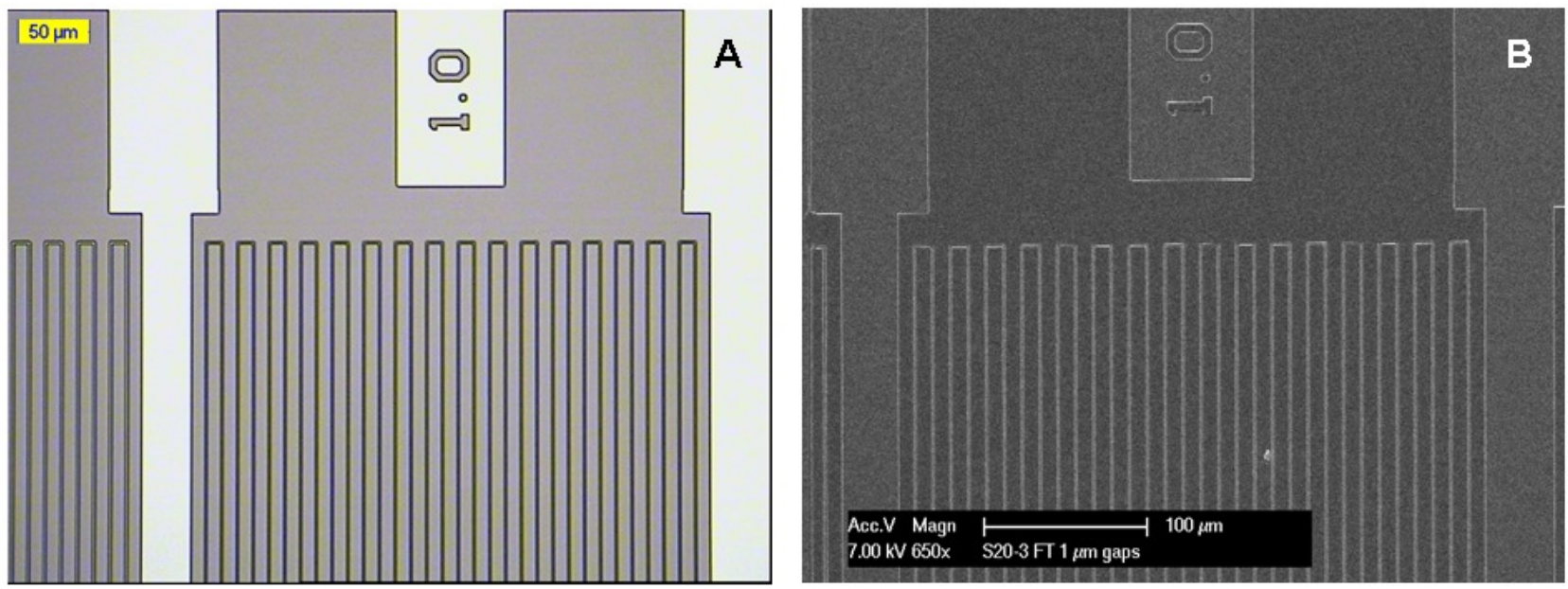

Fig. 6. Optical microscope image of S/D electrodes after development of exposed HPR 504 resist (A), and SEM image of the $200 \mathrm{~nm}$ deep etched Si template (B). The space between electrodes (channel length) is $1.0 \mu \mathrm{m}$.
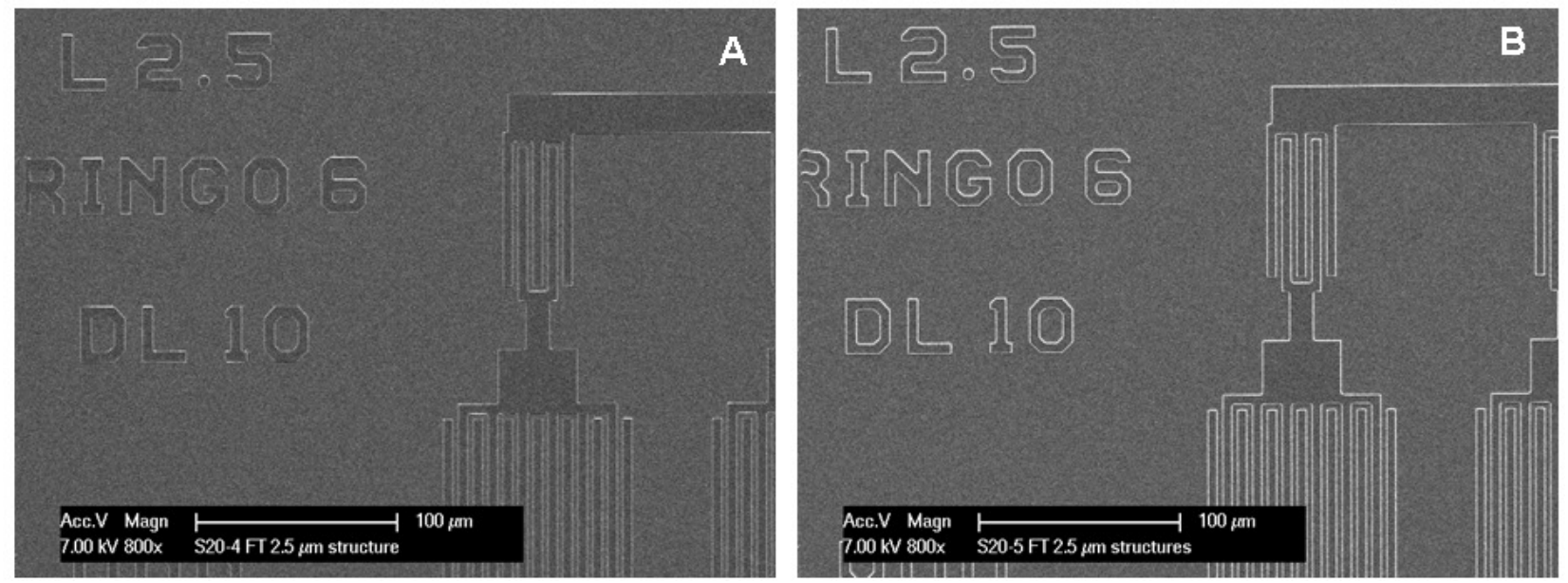

Fig. 7. SEM images of $2.5 \mu \mathrm{m} \mathrm{S} / \mathrm{D}$ electrodes template on Si with etch depth of 400 (A) and 600 (B) $\mathrm{nm}$.

With a typical rotation frequency of $50 \mathrm{~Hz}$ and a track pitch of $200 \mathrm{~nm}$, the writing time of S/D electrodes templates was 34 minutes. 


\subsection{Template manufacturing for a biosensor}

Conventional optical lithography has been previously used for patterning metal layers on plastic substrates such as PEN or PET, and eventually to manufacture functional transistors.[3] Disposable biosensors are another potential application of plastic electronics, and optical lithograph was employed for manufacturing dual detection DNA sensors on PEN foil. The disposable PEN cartridge contains metal electrodes with a $20 \mu \mathrm{m}$ gap, see Fig. 8 A and B. The circuit is closed and a current and an optical signal are read when the captured DNA is attached to the area between the two electrodes. DLW was used to manufacture Si templates having the pattern of the metal electrodes for HE imprint lithography. Optical microscope images of the developed resist deposited on Si wafer are displayed in Fig. 8 A and B (enlarged electrode gap). Reactive ion etching of the exposed $\mathrm{Si}$ under $\mathrm{CF}_{4}$ plasma resulted in templates with various depths $(180-500 \mathrm{~nm})$. A SEM image of the electrode gap on the Si template (Fig. 8C) proves that the image of the developed resist was transferred with fidelity to the underlying wafer, and an electrode gap of $20.14 \mu \mathrm{m}$ was obtained on the template, while the target value was $20.0 \mu \mathrm{m}$.
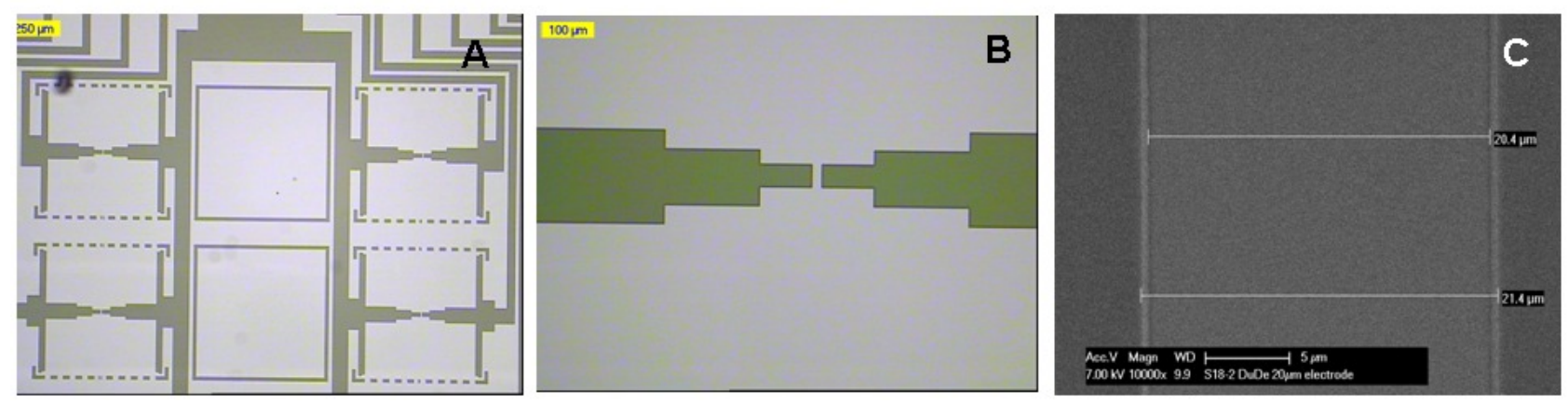

Fig. 8. Optical microscope images of photoresist profile of corresponding to the electrodes of the biosensor (A), (B), and SEM image of the electrodes gap on the Si template after dry etching $(\mathrm{C})$.

The written area was between radii 0.01 and $36.1 \mathrm{~mm}$, for a total writing time of 60 minutes.

\section{TEMPLATE EVALUATION}

The Si templates were evaluated with two types of replication experiments, namely conventional hot embossing of an imprint resist and subsequent pattern of a metal layer, and the direct patterning of a PET foil via hot embossing.

Replication was done in PET foil at a temperature of $140^{\circ} \mathrm{C}$, pressure 40 bar for seven minutes, followed by demolding at $65^{\circ} \mathrm{C}$. Optical microscope images of the replicated surface show that accurate structures were replicated in the PET foil with templates having $180 \mathrm{~nm}$ and $500 \mathrm{~nm}$ depths, see Fig. 9. The gap between the electrodes was measured to be $18.16 \mu \mathrm{m}$.
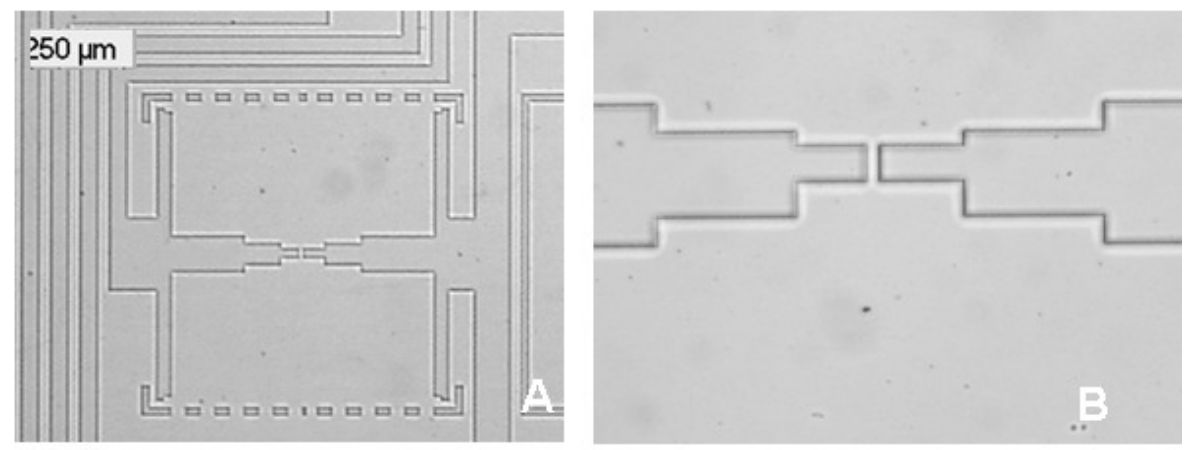

Fig. 9. Optical microscope images of biosensor electrodes replicated in poly(ethyleneterephthalate) foil. Template characteristics: 4 " Si, $180 \mathrm{~nm}$ depth (A), and $500 \mathrm{~nm}$ depth (B).

Metal patterns obtained with a Si template on PEN foil are displayed in Figure 10. On a polished Si wafer a $30 \mathrm{~nm}$ thick $\mathrm{Au}$ layer was deposited. Prior to Au deposition, a $4 \mathrm{~nm}$ Ti layer had been deposited in order to enhance Au adhesion. A 
$620 \mathrm{~nm}$ thick poly(methylmeyhacrylate) layer was deposited by spin-coating on top of the Au layer. HE imprint lithography was performed at $190{ }^{\circ} \mathrm{C}$, a pressure of 40 bars for $300 \mathrm{~s}$ on an Obducat tool. Following reactive ion etching for removal of residual layer, and wet etching, the Au patterns displayed in Figure 10 were obtained.
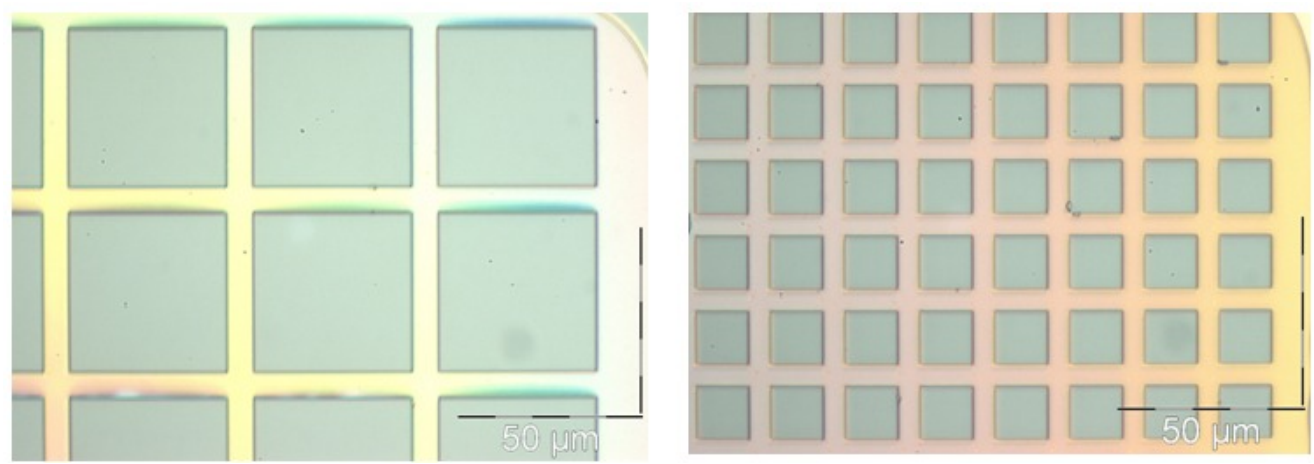

Fig. 10. Optical microscope images of Au patterns obtained with $600 \mathrm{~nm}$ etch depth Si template.

\section{CONCLUSIONS}

DLW is a versatile technology, allowing fast fabrication of templates bearing various structures with dimensions varying from nanometer to micrometer regime. An ideal tool for fast prototypes and templates manufacturing, DLW allows easy and fast modifications of the pattern to be written. The low throughput of this technique is compensated when combined with NIL, which has the potential of being implemented in a roll-to-roll line for fabrication of high volume large area flexible electronics.[1]

By using a laser beam recorder with a wavelength of $405 \mathrm{~nm}$, two types of NIL templates were manufactured, characterized, and tested within the current study. We demonstrated successful manufacturing of silicon templates with source and drain electrodes for transistors on flexible substrates, and patterns of circuits to be incorporated in disposable biosensors. The feature sizes of the templates for S/D electrodes varied from $0.75 \mu \mathrm{m}$ to $>50 \mu \mathrm{m}$, as noticed in the optical microscopy and SEM images. Testing of the templates with layout of a biosensor showed that structures were imprinted on PET substrates with high fidelity. Au structures were patterned with fidelity employing the 4" Si test templates, while experiments are being performed in order to obtain S/D metal electrodes. Exposure with a $266 \mathrm{~nm}$ laser are being performed as well, preliminary results showing $100.0 \mathrm{~nm}$ feature sizes clearly defined.

\section{ACKNOWLEDGEMENTS}

The research work was performed in the framework of the Holst Centre-TNO (The Netherlands). We gratefully acknowledge the contribution of the Holst Centre partners Philips, ASML, and Polymer Vision to the work described in this paper. Mesa+ is acknowledged for their contribution to $\mathrm{Si}$ template evaluation, Marco Barink is gratefully acknowledged for his contribution to the pattern transformation software, and Maria Peter, Arnaud Dauendorffer, and Pieter Moonen for their contribution in the testing of templates. 


\section{REFERENCES}

[1] S. H. Ahn and L. J. Guo, "High-speed roll-to-roll nanoimprint lithography on flexible plastic substrates", $A d v$. Mat. 20 (11), 2044-2049 (2008)

[2] L. J. Guo, "Nanoimprint Lithography: Methods and Material Requirements", Adv. Mat. 19 495-513 (2007)

[3] W. J. M. de Laat, M. Peter, F. Furthner, P. Giesen, C.-Q. Gui and E. R. Meinders, "Submicron patterning on flexible substrates by reduction optical lithography", Proc. SPIE 6921 69212F-69211 - 69212F-69212 (2008)

[4] S.-Y. Hwang, S.-H. Hong, H.-Y. Jung and H. Lee, "Fabrication of roll imprint stamp for continuous UV roll imprinting process", Microel. Eng. in press (2008)

[5] T.-C. Lin, S.-C. Yu, P.-S. Chen, K.-Y. Chi, H.-C. Pan and C.-Y. Chao, "Fabrication of alignment layer free flexible liquid crystal cells using thermal nanoimprint lithography", Curr. Appl. Phys. 9 610-612 (2009)

[6] T. Mäkelä, T. Haatainen, P. Majander and J. Ahopelto, "Continuous roll to roll nanoimprinting of inherently conducting polyaniline", Microel. Eng. 84 877-879 (2007)

[7] G. Leising, B. Stadlober, U. Haas, A. Haase, C. Palfinger, H. Gold and G. Jakopic, "Nanoimprinted devices for integrated organic electronics", Microel. Eng. 83 831-838 (2006)

[8] S. Logothetidis, "Flexible organic electronic devices: Materials, process and applications", Mat. Sci. Eng. B 152 96-104 (2008)

[9] B. Cui, Y. Cortot and T. Veres, "Polyimide nanostructures fabricated by nanoimprint lithography and its applications", Microel. Eng. 83 906-909 (2006)

[10] H. Lee, S. Hong, K. Yang and K. Choi, "Fabrication of nano-sized resist patterns on flexible plastic film using thermal curing nano-imprint lithography", Microel. Eng. 83 323-327 (2006)

[11] W.-c. Liao, S. L.-C. Hsu, S.-Y. Chu and P.-C. Kau, "Imprint lithography for flexible transparent plastic substrates", Microel. Eng. 75 145-148 (2004)

[12] J. A. Lewis and G. M. Gratson, "Direct writing in three dimensions", Mat. Today July/ August 32-39 (2004)

[13] H. J. Booth, "Recent applications of pulsed lasers in advanced materials processing", Thin Solid Films 453-454 450-457 (2004)

[14] M. Flury, A. Benatmane, P. Gerard, P. C. Montgomery, J. Fontaine, T. Engel, J. P. Schunck and E. Fogarassy, "Excimer laser ablation lithography applied to the fabrication of diffractive optics", Appl. Surf. Sci. 208-209 238-244 (2003)

[15] D. Radtke and U. D. Zeitner, "Laser-lithography on non-planar surfaces", Optics Express 15 (3), 1167-1174 (2007)

[16] M. Schrödner, R.-I. Stohn, K. Schultheis, S. Sensfuss and H.-K. Roth, "Polymer field effect transistors made by laser patterning", Org. Electr. 6 161-167 (2005)

[17] A. Pique, C. B. Arnold, C. Wartena, B. Pratap, B. Shashishekar, K. E. Swider-Lyons, D. W. Weir and R. A. Kant, "Laser direct-write of miniature sensor and microbattery systems", Riken Review 50 57-62 (2003)

[18] C. Rensch, S. Hell, M. v. Schickfus and S. Hunklinger, "Laser scanner for direct writing lithography", Appl. Optics 28 (17), 3754-3758 (1989)

[19] E. Forsen, P. Carlberg, L. Montelius and A. Boisen, "Laser lithography on resist bilayer for nanoelectromechanical systems prototyping", Microel. Eng. 73-74 491-495 (2004) 